\title{
NONTORAL PINCHED ANOSOV MAPS
}

\author{
DAVID FRIED ${ }^{1}$
}

\begin{abstract}
We show Anosov maps satisfying a certain spectral property can arise on nilmanifolds of any nilpotent degree, settling a conjecture of Brin.
\end{abstract}

Brin studied Anosov maps on closed Riemannian manifolds satisfying a certain condition on the spectrum of the operator induced on vector fields [3]. He showed that such an $A: M \rightarrow M$ is transitive and that $M$ is covered by Euclidean space and he conjectured that $M$ must be a torus. We show that, on the contrary, Anosov automorphisms satisfying Brin's condition may be constructed on nilmanifolds of any nilpotent degree.

This result for degree 2 is joint work with S. Dani and we were helped greatly by conversations with R. Szczarba. We also thank Yale University for supporting this research.

Dani has pointed out that the examples constructed below for degree $>2$ are new examples of Anosov diffeomorphisms.

Brin's condition is that the spectrum of the operator $A_{*}: X(M) \rightarrow X(M)$ defined by $\left(A_{*}(v)\right)(m)=D A\left(A^{-1} m\right)(v(m))$ lies in the union of two annuli $\left\{x \in \mathrm{C} \mid 0<r_{1}<\right.$ $|x|<r_{2}<1$ or $\left.1<R_{2} \leqslant|x|<R_{1}<\infty\right\}$ satisfying

$$
1+\frac{\log R_{2}}{\log R_{1}}>\frac{\log r_{1}}{\log r_{2}}
$$

By work of Mather [6], $A$ is Anosov iff the spectrum of $A_{*}$ lies off the unit circle; if (*) holds, we will call $A$ pinched.

The construction is based on the scheme of Auslander and Scheuneman [1]. Given $d$ we will construct a semisimple matrix $A_{1} \in \operatorname{SL}(d, Z)$ with special eigenvalues. This $A_{1}$ extends to a Lie automorphism $A_{k}$ of the free $k$-step nilpotent Lie algebra with $d$ generators $N_{k}\left(\mathbf{R}^{d}\right)$. For a certain ideal $I, A_{k}$ induces a lattice-preserving automorphism $\bar{A}$ of $N_{k}\left(\mathbf{R}^{d}\right) / I$. If $k<\sqrt{d}$, this map $\bar{A}$ induces a pinched Anosov automorphism $A$ on a nilmanifold of nilpotent degree exactly $k$.

1. The construction of $A_{1}$. Let $d>2$ be an integer. Let $F$ be a totally real algebraic number field of degree $d$ over $Q$. By the geometric version of the Dirichlet Unit Theorem [2], the map $l: F^{*} \rightarrow\left\{\left(y_{i}\right) \in \mathbf{R}^{d} \mid \Sigma y_{i}=0\right\}=V, l(x)=$ $\left(\log \left|x^{(i)}\right|\right)$ where $x=x^{(1)}, x^{(2)}, \ldots, x^{(d)}$ are the conjugates of $x$, carries the algebraic units of $F$ onto a lattice $L_{0} \subset V$. By going far out along the ray through

Received by the editors August 6, 1980.

1980 Mathematics Subject Classification. Primary 58F15; Secondary 22E40.

${ }^{1}$ Partially supported by the National Science Foundation. 
$(1-d, 1, \ldots, 1) \in V$ and choosing a nearby lattice point $l(x)$, one obtains a unit $x$ satisfying $|\log | x^{(i)}|/ \log \lambda-1|<\varepsilon$ for some $\lambda>1, i=2, \ldots, d$, where $\varepsilon=\varepsilon(d)$ will be chosen later.

The unit $x$ acts on $\mathbf{R}^{d}$ and preserves the lattice $L_{0}$ via $\left(z^{(i)}\right) \mapsto\left(x^{(i)} z^{(i)}\right)$. Let $A_{1} \in \mathrm{SL}(d, \mathrm{Z})$ be the matrix representing this action relative to an integral basis for $L_{0}$. Clearly the original coordinate directions are eigenvectors for $A_{1}$ with eigenvalues $x^{(1)}, \ldots, x^{(d)}$, so $A_{1}$ is semisimple. Moreover, the property that $\left|x^{(i)}\right| \approx \lambda>1$ for $i>1$ implies that the characteristic equation of $A_{1}, \Pi_{i}\left(t-x^{(i)}\right)$, is irreducible. Hence the $x^{(i)}$ are distinct. The eigenvector $e_{1}$ corresponding to $x^{(1)}$ may be chosen to have coordinates in $\mathbf{Q}\left(x^{(1)}\right)$. Conjugation of these coordinates gives eigenvectors $e_{2}, \ldots, e_{d}$ corresponding to the eigenvalues $x^{(2)}, \ldots, x^{(d)}$.

2. Construction of the Lie automorphism $\bar{A}$. For $k>$ ?, let $N_{k}\left(\mathbf{R}^{d}\right)$ be the free $k$-step nilpotent Lie algebra on $d$ generators. Let $I=I\left(A_{1}\right) \subset N_{k}\left(\mathbf{R}^{d}\right)$ be the vectorspace spanned by all products of eigenvectors $e_{i}$ in which some $e_{i}$ appears at least twice. Clearly $I$ is an ideal and is invariant under the Lie automorphism $A_{k}$ of $N_{k}\left(\mathbf{R}^{d}\right)$ obtained by extending the action of $A_{1}$ on $\mathbf{R}^{d}$.

We will show that, relative to the natural integral lattice $L_{k} \subset N_{k}\left(\mathbf{R}^{d}\right), I$ is spanned by integral vectors. Clearly $I$ is spanned by those products consisting of 2 copies of $e_{i}$, some $i=1, \ldots, d$, and the other factors integral vectors. Fixing a choice of integral vectors and an order in which the multiplication is to be performed and letting $e_{i}$ vary over the eigenbasis chosen in $\$ 1$ gives vectors $s_{1}, \ldots, s_{d} \in I$. Note that $s_{1}$ is defined over $\mathbf{Q}\left(x^{(1)}\right)$, so $s_{1}=\sum_{p=0}^{d-1}\left(x^{(1)}\right)^{p} v_{p}$ where $v_{p}$ is a rational vector. Since the $e_{i}, i>1$, are conjugates of $e_{1}$, we have $s_{i}=$ $\sum_{p=0}^{d-1}\left(x^{(i)}\right)^{p} v_{p}$. The transformation $\left(\left(x^{(i)}\right)^{p}\right)$ carrying $\left(v_{p}\right)_{p=0}^{d-1}$ to $\left(s_{i}\right)_{i=1}^{d}$ is invertible, since it is a Vandermond matrix and the $x^{(i)}$ are distinct. Thus the span of the $s_{i}$ equals the span of the $v_{p}$ and $I$ is spanned by rational vectors $v_{p}$.

Thus $L_{k}$ meets $I$ in a sublattice of rank equal to $\operatorname{dim} I$, and so the integral vectors in $\bar{N}=N_{k}\left(\mathbf{R}^{d}\right) / I$ form a lattice $\Gamma$. It is clear that $A_{k}$ induces an automorphism $\bar{A}$ of $\bar{N}$ which preserves $\Gamma$.

3. Hyperbolicity and pinching. We show that for the proper choice of $\varepsilon(d)$ and for $k<\sqrt{d}$ that $\bar{A}$ is hyperbolic and its eigenvalues satisfy the pinching condition. Once these facts are shown, it will follow that $\bar{A}$ induces a hyperbolic automorphism $\tilde{A}$ of the simply connected Lie group $G$ determined by $\bar{N}$. The theory of Malcev [5] implies that $\exp \left(\Gamma^{\prime}\right)$ is a lattice, for some $\Gamma^{\prime}$ of finite index in $\Gamma$. Since the lattices $m L_{1}, m \geqslant 1$, generate a cofinal collection $\Gamma_{m}^{\prime}$ of sublattices of $\Gamma$, we may assume $\Gamma^{\prime}=\Gamma_{m}^{\prime}$. But since $\Gamma_{m}^{\prime}$ is isomorphic to $\Gamma=\Gamma_{1}^{\prime}$ under the automorphism of $\bar{N}$ induced by multiplication by $m$ on $\mathbf{R}^{d}$, it must be that $\exp (\Gamma)$ is itself a lattice. Hence $\bar{A}$ induces an automorphism $A$ of $M=G / \exp (\Gamma)$. Using the parallelization on $M$ by left-invariant vector fields, it is easy to check that the pinching condition on the eigenvalues of $\bar{A}$ implies that $A$ is pinched.

The eigenvectors (resp. eigenvalues $\mu$ ) of $\bar{A}$ are all products of $<k$ distinct eigenvectors $e_{i}$ (resp. eigenvalues $x^{(i)}$ ), by our choice of the ideal $I$. Thus one may 
estimate, according to whether $e_{1}$ occurs or not,

$$
\log r_{1}=\log \left|x^{(1)}\right|<\log |\mu|<\log \left|x^{(1)}\right|+(k-1) \sup \log \left|x^{(i)}\right|=\log r_{2}
$$

or

$$
\log R_{2}=\inf _{i>1} \log \left|x^{(i)}\right|<\log |\mu|<k \cdot \sup \log \left|x^{(i)}\right|=\log R_{1} .
$$

Taking logs to the base $\lambda$ and using $d>k^{2}$, one has

$$
\left\{\begin{array}{l}
\log r_{2} \approx-(d-k)<0 \\
\log R_{2} \approx 1>0
\end{array}\right.
$$

and

$$
\left\{\frac{\log r_{1}}{\log r_{2}} \approx \frac{-(d-1)}{-(d-k)}<1+\frac{1}{k} \approx 1+\frac{\log R_{2}}{\log R_{1}} .\right.
$$

So for $\varepsilon(d)$ sufficiently small, $\bar{A}$ is hyperbolic and the desired pinching condition holds.

Finally we must check that the $k$ th term $C^{k}(\bar{N})$ in the central series of $\bar{N}$ is nontrivial. However, it is clearly spanned by all products of $k$ distinct eigenvectors from the eigenbasis $e_{1}, \ldots, e_{d}$. By $[4,11.2 .4]$ one may pick a basis by choosing any $k$ distinct $e_{i}$ in any cyclic order and introducing brackets in a standard way. It follows that $C^{k}(\bar{N})$ has dimension $\left(\begin{array}{c}d \\ k\end{array}\right) \cdot(k-1)$ !, which is positive for $d>k$.

Thus for $d>k^{2}$ all the desired properties hold.

\section{References}

1. Louis Auslander and John Scheuneman, On certain automorphisms of nilpotent Lie groups, Global Analysis, Proc. Sympos. Pure Math., vol. 14, Amer. Math. Soc., Providence, R. I., 1968.

2. Z. I. Borevich and I. R. Shafarevich, Number theory, Academic Press, New York, 1966.

3. M. I. Brin, Nonwandering points of Anosov diffeomorphisms, dynamical systems. I, Asterisque, Warsaw, vol. 49, 1977.

4. Marshall Hall, The theory of groups, Macmillan, New York, 1959.

5. A. Malcev, On a class of homogeneous spaces, Amer. Math. Soc. Transl. 39 (1951).

6. John N. Mather, Characterization of Anosov diffeomorphisms, Nederl. Akad. Wetensch. Indag. Math. 30 (1968), 479-483.

Deparmient of Mathematics, University of California, Santa Cruz, California 95064 